\section{Lack of an association between blood genotype and haemoplasma infection in UK cats}

\author{
Sarah Spencer', Chris Helps', \\ Richard Malik ${ }^{2}$, Barbara Gandolfi ${ }^{3}$, \\ Séverine Tasker ${ }^{1}$

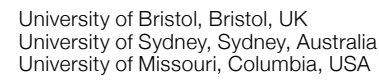

In humans, examples exist where blood type provides protection against infectious diseases, for example susceptibility to malaria caused by the haemotrophic Plasmodium species, and Helicobacter pylori, norovirus and cholera infections. Many blood type antigens are receptors for pathogenic microorganisms and it is proposed that pathogens played an important role in the evolution of blood type polymorphism by providing an evolutionary advantage.

Blood type frequencies differ between domestic feline populations, as they do in humans. The genetic basis of the feline $A B$ blood group system has been recently investigated, with blood type being determined by variants in the cytidine monophospho- $\mathrm{N}$-acetylneuraminic acid hydroxylase $(C M A H)$ gene. Haemoplasma infection is common in cats worldwide and can cause significant anaemia. The aim of this study was to determine whether any association existed between blood genotype and feline haemoplasma infection status in cats from the UK.

Blood genotype ( $A A, A b$ or $b b)$ was determined by pyrosequencing two variants in exon 2 of $C M A H$ in 263 cats that had previously tested positive $(n=131)$ or negative $(\mathrm{n}=132)$ for Mycoplasma haemofelis, 'Candidatus Mycoplasma haemominutum', and 'Candidatus Mycoplasma turicensis' infection by quantitative PCR. Possible associations between genotype and haemoplasma infection status were investigated by $\mathrm{x} 2$ analysis. Significance was taken as $\mathrm{P}<0.05$.

Blood genotyping revealed 122 (46\%) AA, 104 (40\%) Ab and $37(14 \%) b b$ cats. Of those that tested positive for one or more haemoplasma species, 56 (43\%) were $A A, 60$ (46\%) were $A b, 15$ (11\%) were $b b$. Of those that tested negative, 66 (50\%) were $A A, 44$ (33\%) were $A b, 22$ (17\%) were bb. No significant difference in the prevalence of haemoplasma infection between blood genotypes was found $(x 2=4.602$, $p=0.10)$. The prevalence of each individual haemoplasma species, single versus dual haemoplasma species infection, and haemoplasma PCR copy number, were also compared between blood genotypes and no significant differences were found.

The hypothesis that haemoplasma infection prevalence was associated with blood genotype was not supported in this study. To the authors' knowledge, this is the first study investigating the relationship between blood type and infectious disease prevalence in cats and further studies investigating other infectious diseases, particularly those that infect feline erythrocytes, are warranted. 